\title{
How should we treat air leaks?
}

\author{
Ikenna Okereke, MD, FACS
}

See related article on pages $982-8$.

Air leak is a common and occasionally challenging problem for thoracic surgeons after pulmonary resection. Many different treatment options have been attempted, more recently including resorbable hydrogels and endobronchial valve placement. In their study in this issue of the Journal, Tsubokawa and colleagues ${ }^{1}$ analyze a canine model in which TachoSil (Nycomed GmbH, Linz, Austria), a fibrin sealant patch, is placed over an area of the canine lung that was injured so as to create an air leak. The lung parenchyma in these animals was normal, however, in contrast to the emphysematous lung parenchyma in most patients with lung cancer. A model that induced emphysematous changes in the animal model would more closely mimic clinical challenges faced by thoracic surgeons and thus yield a more relevant study. Clearly, patients with more advanced emphysematous changes are more likely to have air leaks develop after surgery and are more likely to have a persistent air leak. It is expected that a patient with significant emphysematous changes (Figure 1, $A$ ) would be more likely to have a persistent air leak than would a patient with minimal emphysematous changes (Figure 1, B). TachoSil application might have different efficacies in these patients

From the Division of Thoracic Surgery, Warren Alpert Medical School of Brown University, Providence, RI.

Disclosures: Author has nothing to disclose with regard to commercial support.

Received for publication Jan 8, 2015; accepted for publication Jan 10, 2015; available ahead of print Feb 11, 2015.

Address for reprints: Ikenna Okereke, MD, FACS, Division of Thoracic Surgery, The Rhode Island and Miriam Hospitals, Warren Alpert Medical School of Brown University, 2 Dudley St, Suite 470, Providence, RI 02903 (E-mail: iokereke@ usasurg.org).

J Thorac Cardiovasc Surg 2015;149:960-1

$0022-5223 / \$ 36.00$

Copyright $(\underset{2}{ } 2015$ by The American Association for Thoracic Surgery

http://dx.doi.org/10.1016/j.jtcvs.2015.01.017 and might also be associated with different histologic changes than seen in lung parenchyma without significant emphysema.

Previous in vivo animal models for air leak have been attempted. ${ }^{2}$ The methods to induce air leak have ranged from focal injury to pulmonary resection with abrasion of the fissure line. In this study, a focal injury measuring $1 \times 1$ $\times 0.2 \mathrm{~cm}$ was created. The overall depth of this injury did not mimic the usual clinical air leak, which is deeper, covers a broader area, and occurs in emphysematous lung parenchyma. It is quite possible that these air leaks would have healed without administration of TachoSil. Furthermore, the histologic changes that occurred might have been altered in the presence of a more significant air leak. A model that mimicked the type of injury most often encountered by thoracic surgeons would be preferable.

The number of TachoSil patches used did not appear to be uniform. Furthermore, with such a small defect created as the model for air leak, it is unclear why more than 1 patch needed to be used per defect, or 2 per animal. And if 3 to 4 TachoSil patches are needed for such a focal defect, how many TachoSil patches would be needed in the usual clinical scenario encountered by thoracic surgeons? When analyzing newer agents to use to treat air leaks, a costbenefit breakdown must be a part of the overall analysis. ${ }^{3}$ Given the number of TachoSil patches needed for the injuries in this study, it does not appear that TachoSil patches would be a feasible or cost-effective method to treat air leak clinically.

In their article, Tsubokawa and colleagues ${ }^{1}$ mention that the animals could not have survived with an uncontrolled air leak. They also mention that the experimental animals had no air leak observed as they closed the chest. In practice, however, it can be quite difficult to appreciate small air leaks, and they often occur over a broad area, such as a fissure line after lung resection. In addition, in the model created by Tsubokawa and colleagues ${ }^{1}$ there is no space

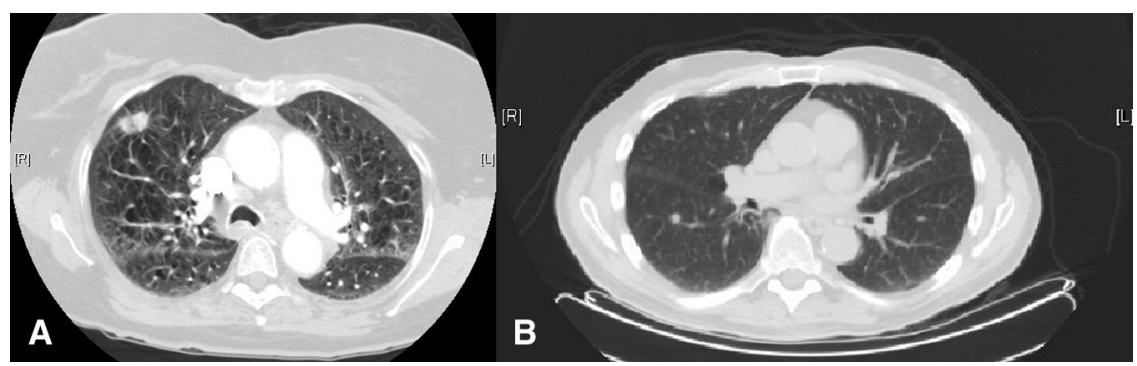

FIGURE 1. Patient with significant emphysematous changes (A) and patient with minimal emphysematous changes (B). 
in the chest. The combination of a superficial, focal wound used to create the air leak model, lack of a space in the chest, and nonemphysematous lungs makes clinical translation and relevance difficult to ascertain.

I commend Tsubokawa and colleagues ${ }^{1}$ for attempting to create an animal model to mimic air leak and for examining a novel approach to treating air leak. From this study it appears that TachoSil patches can be used safely and without significant inflammation to the lung tissue. Two questions, however, are not answered by this study: (1) Are TachoSil patches effective in preventing air leak after lung resection, particularly in emphysematous lungs? (2) Is application of a
TachoSil patch feasible after lung resection, especially in multiple areas or over a broad surface of lung? This report should stimulate future studies into different models that more closely reproduce the typical clinical scenario.

\section{References}

1. Tsubokawa N, Miyata Y, Mimae T, Sasada S, Yoshiya T, Mimura T, et al. Histologic changes associated with the use of fibrinogen and thrombin-impregnated collagen in the prevention of pulmonary air leakage. J Thorac Cardiovasc Surg. 2015;149:982-8.

2. Kobayashi H, Sekine T, Nakamura T, Shimizu Y. In vivo evaluation of a new sealant material on a rat lung air leak model. J Biomed Mater Res. 2001;58:658-65.

3. Mahajan AK, Doeing DC, Hogarth DK. Isolation of persistent air leaks and placement of intrabronchial valves. J Thorac Cardiovasc Surg. 2013;145:626-30. 